\title{
Photoelectrochemistry of Photosystem II in Vitro vs in Vivo
}

\author{
Jenny Z. Zhang, ${ }^{\dagger}$ Paolo Bombelli, ${ }^{\ddagger}$ Katarzyna P. Sokol, ${ }^{\dagger}$ Andrea Fantuzzi, ${ }^{\S}$ A. William Rutherford, ${ }^{\S}$ \\ Christopher J. Howe, ${ }^{+}$and Erwin Reisner* ${ }^{\dagger}$ (1)
}

${ }^{\dagger}$ Department of Chemistry, University of Cambridge, Cambridge CB2 1EW, United Kingdom

${ }^{\ddagger}$ Department of Biochemistry, University of Cambridge, Cambridge CB2 1QW, United Kingdom

${ }^{\S}$ Department of Life Sciences, Imperial College London, London SW7 2AZ, United Kingdom

Supporting Information

ABSTRACT: Factors governing the photoelectrochemical output of photosynthetic microorganisms are poorly understood, and energy loss may occur due to inefficient electron transfer (ET) processes. Here, we systematically compare the photoelectrochemistry of photosystem II (PSII) protein-films to cyanobacteria biofilms to derive: (i) the losses in light-to-charge conversion efficiencies, (ii) gains in photocatalytic longevity, and (iii) insights into the ET mechanism at the biofilm interface. This study was enabled by the use of hierarchically structured electrodes, which could be tailored for high/stable loadings of PSII core complexes and Synechocystis sp. PCC 6803 cells. The mediated photocurrent densities generated by the biofilm were 2 orders of magnitude lower than those of the protein-film. This was partly attributed to a lower photocatalyst loading as the rate of mediated electron extraction from PSII in vitro is only double that of PSII in vivo. On the other hand, the biofilm exhibited much greater longevity ( $>5$ days) than the protein-film $(<6 \mathrm{~h})$, with turnover numbers surpassing those of the protein-film after 2 days. The mechanism of biofilm electrogenesis is suggested to involve an intracellular redox mediator, which is released during light irradiation.

$\mathrm{O}$ xygenic photosynthetic microorganisms, such as cyanobacteria and algae, have evolved adaptive mechanisms to harness solar energy and thrive in a diverse range of habitats. ${ }^{1}$ Underpinning this is the light-driven water oxidation reaction carried out by the dimeric photosystem II (PSII) complex. ${ }^{2}$ PSII core complexes can be wired to electrodes (Figure S1 for ET mechanism) in photoelectrochemical studies to generate lessons for both natural and artificial photosynthesis., Currently, there are also growing efforts to immobilize photosynthetic microorganisms onto electrodes to perform useful photoelectrochemistry. ${ }^{5-7}$ This is because such organisms are robust, abundant, inexpensive to culture, and capable of performing energy storage/conversions, including photobioelectrogenesis (i.e., light-driven electricity production by living organisms). ${ }^{8,9}$

Although photocurrents of up to $1 \mathrm{~mA} \mathrm{~cm}{ }^{-2}$ have been observed in PSII protein-film systems, ${ }^{10}$ the outputs of photosynthetic biofilm systems are generally much lower $\left(<50 \mu \mathrm{A} \mathrm{cm}^{-2}\right.$, Table S1), despite the employment of mediated regimes and bioengineering efforts. ${ }^{711}$ This low performance may be due to the micro-sized "footprint" of the cells compared to the nanosized enzymes, resulting in substantially lower photocatalyst loading densities on commonly employed flat electrodes. Furthermore, the ET mechanism at the biofilmelectrode interface is poorly understood, though three ET pathways have been put forward (Figure 1). ${ }^{5,12}$ As such, the extent of inefficiency and energy loss by electrons originating from PSII en route to the electrode is also speculative.

A side-by-side comparison in the photoelectrochemistry of PSII in vitro (i.e., protein-films of core complexes) vs in vivo (i.e., photosynthetic biofilms) may provide valuable quantitative assessments into trade-offs in efficiency and longevity, and clues into the ET pathways at the biointerface. However, such a comparison is challenging due to vast differences in electrode designs and experimental methodologies between (and even within) the protein-film and biofilm fields (Table S1).

Herein, we exploit the versatility of state-of-the-art inverse opal indium-tin oxide (IO-ITO) electrodes for protein films, ${ }^{10}$ and synthesized analogous electrodes with adapted dimensions for biofilm integration (Figure $1 \mathrm{~A}-\mathrm{C}$ ). These electrodes allowed us to systematically compare biocatalyst loadings, photoelectrochemical profiles, charge outputs, turnover frequencies (TOF), external quantum efficiencies (EQE), and turnover numbers (TON), between PSII protein-films and cyanobacterial biofilms.

Synechocystis sp. PCC 6803 cells (Synechocystis) were employed in this study as they form biofilms on ITO, ${ }^{8}$ are well-characterized, and are model organisms used in biophotovoltaics. ${ }^{6,13,14}$ PSII core complexes from the cyanobacterium Thermosynechococcus elongatus were employed as they are also well-characterized, relatively robust, and widely studied using protein-film photoelectrochemistry (see SI for more information). ${ }^{4}$

To accommodate the Synechocystis cells (ca. $2 \mu \mathrm{m}$ in diameter, Figure S2), IO-ITO electrodes with an optimized pore size (pore diameter: $10 \mu \mathrm{m}$, channels: $3 \mu \mathrm{m}$ ) and thickness $(40 \mu \mathrm{m})$ for cell loading were developed (Figures S3 and S4). Planktonic cultures of Synechocystis were dropcast onto the IO-ITO electrodes and allowed to mature into a biofilm. A visibly green IO-ITOlbiofilm electrode was obtained after 2-3 days (Figure 1A) and SEM images confirmed cell-cell and cell-electrode adhesion throughout the electrode (Figure S4B). Chl $a$ analysis showed an average loading of $2.5 \pm 0.5$

Received: August 11, 2017

Published: September 15, 2017 


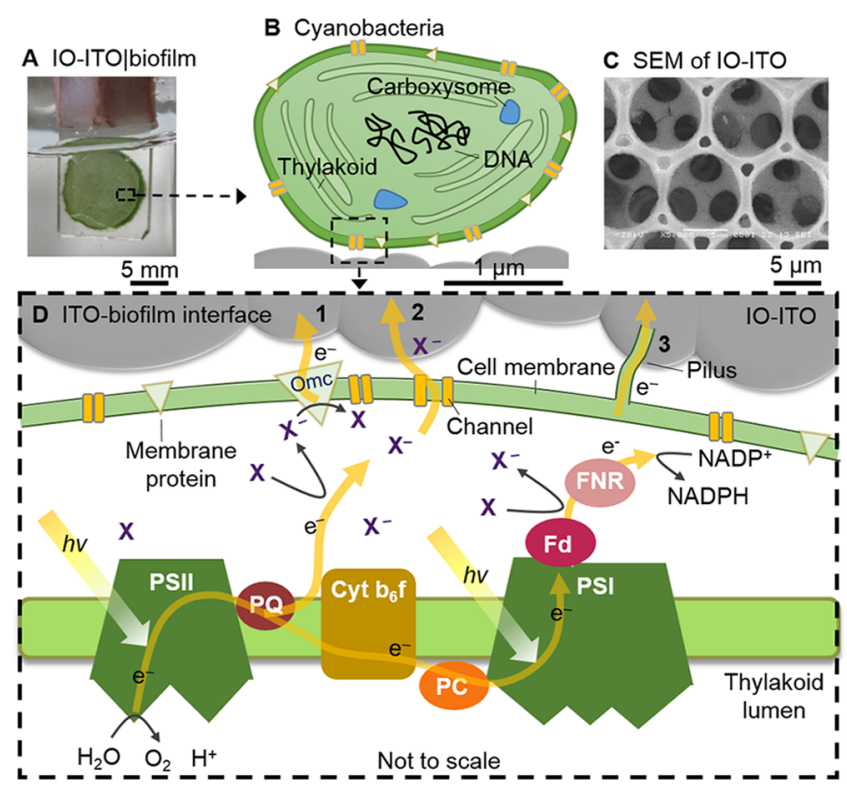

Figure 1. (A) Photograph of an IO-ITO electrode hosting Synechocystis. (B) Scheme of a cyanobacterium on the electrode. (C) Scanning electron microscopy (SEM) image of the IO-ITO electrode. (D) Proposed ET pathways at the biofilm-electrode interface. ${ }^{5,12}$ Pathway 1: direct ET from a membrane-bound redox protein. Pathway 2: indirect ET due to the efflux of intracellular redox species into the extracellular matrix. Pathway 3: indirect ET via pili. X: unknown redox mediator.

$\mu \mathrm{g}$ Chl $a$ per geometrical $\mathrm{cm}^{2}$ for IO-ITOlbiofilm. The opencircuit potential $\left(V_{\mathrm{OCP}}\right)$ of the IO-ITOlbiofilm dropped from ca. $0.3 \mathrm{~V}$ (dark) to below $0.25 \mathrm{~V}$ vs SHE when the electrode was irradiated $\left(\lambda_{685 \mathrm{~nm}}: 1 \mathrm{~mW} \mathrm{~cm}{ }^{-2}\right)$, which is indicative of light-promoted charge movement toward the electrode (Figure S5).

Analogously, PSII dimer complexes $(20 \times 10 \times 10 \mathrm{~nm}$ in dimension) were adsorbed onto $40 \mu \mathrm{m}$ thick IO-ITO electrodes (IO-ITOIPSII) of the same geometric surface area with $750 \mathrm{~nm}$ diameter pores (previously optimized for PSII). ${ }^{10}$ $\mathrm{Chl} a$ analysis showed an average loading of $10.2 \pm 0.4 \mu \mathrm{g} \mathrm{Chl}$ a per (geometrical) $\mathrm{cm}^{2}$ for IO-ITOIPSII. The number of PSII dimers present within each electrode could be calculated based on the Chl $a$ loading. A PSII/PSI ratio within cyanobacteria has been spectroscopically determined to vary from 0.3 to $0.7,{ }^{15}$ and has shown to be lower in Synechocystis. ${ }^{16}$ For simplicity, we assumed a PSII/PSI ratio of 1 in the biofilm systems (see SI for details), which would likely result in a conservative estimation of TOF and TON for the biofilms.

Stepped chronoamperometry scans of the IO-ITOIPSII and IO-ITOlbiofilm electrodes were carried out to analyze their photoresponse profiles as a function of applied potential $\left(E_{\text {app }}\right)$ under chopped light irradiation (Figure 2). An anodic photocurrent onset potential of $0.1 \mathrm{~V}$ was observed in both cases (Figure S6), although a more negative onset potential may have been expected for IO-ITOlbiofilm if the electrons stem from downstream of photosystem I. ${ }^{13}$ However, it is difficult to probe into more negative potential regions due to the generation of trace amounts of $\mathrm{O}_{2}$ at the biofilm-electrode interface, which gives rise to competing charge transfer pathways. ${ }^{17}$ In contrast to IO-ITOIPSII, the amplitude of the photoanodic response by IO-ITOlbiofilm did not increase with increasing $E_{\text {app }}$. This suggests that the rate-limiting ET step in the IO-ITOlbiofilm is not a direct electron injection process
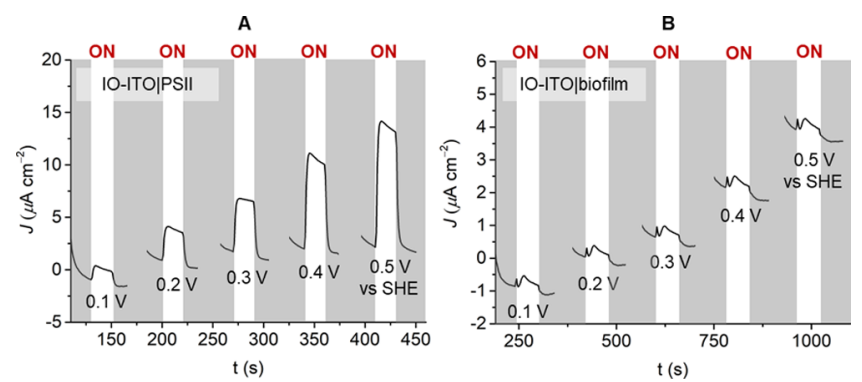

Figure 2. Stepped chronoamperometry scans of (A) IO-ITOIPSII and (B) IO-ITOlbiofilm in the absence of exogenous mediators (DET) and under chopped light irradiation. MES electrolyte solution $(\mathrm{pH}$ 6.5) and BG11 medium ( $\mathrm{pH}$ 8.0) were used in experiments involving IO-ITOIPSII and IO-ITOlbiofilm, respectively. All experiments were carried out under $\operatorname{Ar}$ at $25{ }^{\circ} \mathrm{C}$, using a red light source $\left(\lambda_{685 \mathrm{~nm}}: 1 \mathrm{~mW}\right.$ $\left.\mathrm{cm}^{-2}\right)$.

(see below for further discussion). Further comparisons between the two electrode systems were performed at their respective highest photoresponse at the most negative $E_{\text {app }}(0.3$ $\mathrm{V}$ for IO-ITOlbiofilm; $0.5 \mathrm{~V}$ for IO-ITOIPSII), which is also the potential displaying maximum energy efficiency.

Comparison of the initial direct ET (DET, i.e., absence of exogenous mediators) photoresponse of the two systems showed that IO-ITOIPSII exhibited $>30$-fold higher photocurrent density and EQE than that of IO-ITOlbiofilm (Table 1). However, the 4-fold higher $\mathrm{Chl} a$ loading in the IO-ITOI PSII electrode compared to the IO-ITOlbiofilm should be taken into account. On the basis of the Chl $a$ loading, we calculated ca. 20-fold less PSII dimers in IO-ITOlbiofilm (7.8 \pm $1.6 \mathrm{pmol}$ per geometrical $\left.\mathrm{cm}^{2}\right)$ than in IO-ITOIPSII $(163 \pm 7$ pmol per geometrical $\mathrm{cm}^{2}$ ). When normalized by the amount of PSII present, the TOF of IO-ITOIPSII was approximately thrice that of IO-ITOlbiofilm.

Addition of 2,6-dichloro-1,4-benzoquinone (DCBQ) to the electrolyte solution gave rise to mediated ET (MET) from PSII that was unable to donate electrons to the electrode surface directly (e.g., due to misorientation or spatial separation). The mediated biofilm exhibited maximum photoresponse at $0.5 \mathrm{~V}$ (Figure S6C), corresponding to an enhancement by more than 1 order of magnitude (Table 1). The TOF of IO-ITOIPSII (MET) was approximately twice that of IO-ITOlbiofilm (MET). As such, the photocatalytic turnover rate of PSII in vivo vs in vitro is comparable. This infers that the lower overall photocurrent output of the biofilm system is partly due to the lower density of the photoabsorber/PSII in the IO-ITOlbiofilm electrode. The mediated TOF of the PSII protein-film is comparable to its TOF in solution (Table S2), suggesting that no significant enzyme inactivation occurs at the electrode interface. Note that this mediated TOF of PSII is lower than its theoretical maximum due to the low light intensity of ca. 35 photons $\mathrm{PSII}^{-1} \mathrm{~s}^{-1}$, which is nonsaturating for the $Q_{B}$ diffusional step (rate limiting step: $10 \mathrm{~ms}){ }^{4}$

Addition of the PSII inhibitor (3-(3,4-dichlorophenyl)-1,1dimethylurea (DCMU) to the electrolyte solution of IO-ITOI biofilm in the absence of exogenous mediators resulted in the ceasing of the photoelectrochemical response (Figure S7A), thereby confirming that the photocurrents stem from water oxidation. ${ }^{13}$ Providing DCBQ to produce the enhanced MET photoresponse before adding DCMU resulted in some remaining activity (Figure S7B), which suggests that the MET photocurrents partially stem from elsewhere. 
Table 1. Summary of IO-ITO|PSII and IO-ITO|Biofilm Photoelectrochemical Performance $\left(\lambda_{685} \mathrm{~nm}, 1 \mathrm{~mW}^{\mathrm{cm}}{ }^{-2}\right)$ at $25{ }^{\circ} \mathrm{C}$

\begin{tabular}{|c|c|c|c|c|}
\hline electrode & $E_{\text {app }}\left(V_{\text {SHE }}\right)$ & $J\left(\mu \mathrm{A} \mathrm{cm}^{-2}\right)$ & TOF $\left(s^{-1}\right)$ & EQE (\%) \\
\hline \multirow[t]{2}{*}{ ITOIPSII (DET) } & 0.3 & $7.0 \pm 0.3$ & $7.2 \pm 0.5 \times 10^{-2}$ & $1.3 \pm 0.1$ \\
\hline & 0.5 & $13.7 \pm 1.3$ & $12.8 \pm 0.5 \times 10^{-2}$ & $2.3 \pm 0.2$ \\
\hline \multirow[t]{2}{*}{ ITOIPSII (MET) } & 0.3 & $164 \pm 8$ & $1.7 \pm 0.1$ & $29.7 \pm 1.5$ \\
\hline & 0.5 & $185 \pm 14$ & $1.9 \pm 0.2$ & $33.5 \pm 2.5$ \\
\hline ITOlbiofilm (DET) & 0.3 & $0.21 \pm 0.04$ & $4.6 \pm 0.9 \times 10^{-2}$ & $3.8 \pm 0.7 \times 10^{-2}$ \\
\hline \multirow[t]{2}{*}{ ITOlbiofilm (MET) } & 0.3 & $8.9 \pm 1.4$ & $8.3 \pm 0.5 \times 10^{-1}$ & $1.6 \pm 0.3$ \\
\hline & 0.5 & $14.7 \pm 0.5$ & $1.1 \pm 0.1$ & $2.7 \pm 0.1$ \\
\hline
\end{tabular}

The photoelectrochemical performance of the IO-ITOI biofilm and IO-ITOIPSII electrode was monitored over 5 days of $12 \mathrm{~h}$ dark-light cycles (Figures 3 and S8). The overall charge generated by the IO-ITOIPSII (MET) before complete inactivation $\left(6 \mathrm{~h}: 3.8 \pm 0.4 \times 10^{-1} \mathrm{C} \mathrm{cm}^{-2}\right)$ greatly surpassed those of IO-ITOlbiofilm (MET and DET) even after several days (Figure 3 inset, Figure S9A). Nevertheless, IO-ITOI biofilm (DET) exhibited increasing photoelectrogenic activity and reached $21 \%$ of the total charge generated by the IO-ITOI PSII by day 5 . The degree of biofilm retention/growth on the electrode was monitored using UV-vis absorption spectroscopy (Figure S10), and was observed to be stable over the 5 days. As such, the TOF and the TON could be calculated over time assuming that the faradaic efficiency for water oxidation in both cases are at unity (Figures 3 and S9). ${ }^{10}$

The TOF of the IO-ITOlbiofilm (DET) was initially low, but gradually increased over the 5 days. Considering that the biofilm was not growing, the increase in photoelectrochemical activity can be attributed to the improvement of electronic connection between the cells and the electrodes over time. The TOF for IO-ITOlbiofilm (MET) was initially much higher, but decreased rapidly and reached near zero after $12 \mathrm{~h}$ (Figure S9B). Correspondingly, the TONs observed for IO-ITOIPSII (MET) and IO-ITOlbiofilm (MET) rose steeply at an early stage (Figures 3 and S9C), but plateaued after $6 \mathrm{~h}$ at $6160 \pm$ 400 and $3220 \pm 360 \mathrm{~mol} \mathrm{O}_{2}$ per mol PSII dimer, respectively. The readdition of fresh DCBQ to the IO-ITOlbiofilm (MET) system did not result in the recovery of initial photoactivity. In an $\mathrm{O}_{2}$ evolution assay employing a Clark-type electrode, the activity of planktonic cyanobacteria was observed to drop following the addition of DCBQ and ferricyanide, suggesting that the mediators are cytotoxic (Table S3). No significant

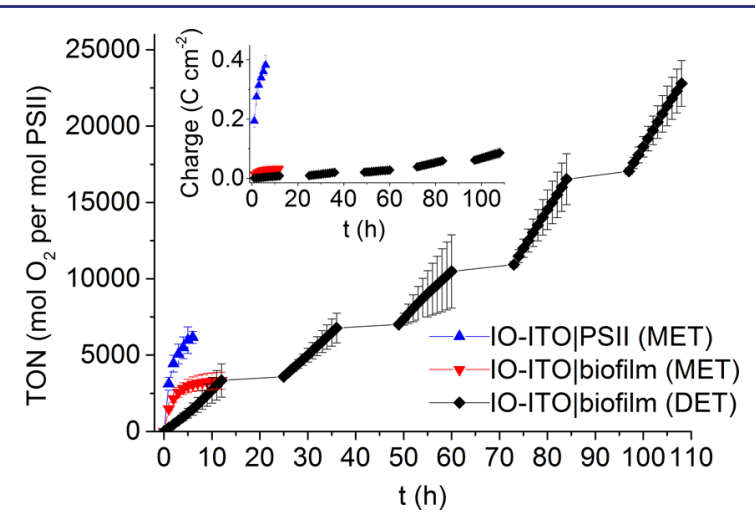

Figure 3. TON of IO-ITOIPSII (MET) and IO-ITOlbiofilm (MET and DET) over 5 days of $12 \mathrm{~h}$ light-dark cycling. Inset: corresponding plot of overall charge passed. Biofilm experiments were carried out under air, and protein-film experiments were carried out under $\mathrm{Ar}$, at $25{ }^{\circ} \mathrm{C}$, using a red light source $\left(\lambda_{685 \mathrm{~nm}}: 1 \mathrm{~mW} \mathrm{~cm}{ }^{-2}\right)$. difference in photoelectrochemical performance resulted from increasing the $E_{\text {app }}$ of the IO-ITOlbiofilm (MET) system to 0.5 $\mathrm{V}$ (Figure S9D) to provide higher driving force for DCBQ oxidation. A detailed summary of these results can be found in Tables S2 and S3.

Although the electron harvesting rate by the electrode is $2-3$ times slower for PSII in vivo than in vitro, PSII in vivo under the DET regime benefits from a considerable gain in photocatalytic longevity. The TON of the IO-ITOlbiofilm (DET) reaches the same TON as the IO-ITOIPSII (MET) within 2 days of $12 \mathrm{~h}$ light irradiation, and is expected to generate the same amount of charge in ca. 25 days.

Clues into the ET mechanism at the biofilm-electrode interface are provided by the photoelectrochemical profile of IO-ITOlbiofilm. From the stepped-chronoamperometry scan of IO-ITOlbiofilm (Figure 2), an indirect ET mechanism in line with pathway 2 depicted in Figure 1D is inferred, whereby the photoresponse is mostly dependent on the available concentration of a diffusional intracellular reduced species. A closer look at the photocurrent profiles reveals further differences between the IO-ITOIPSII and the IO-ITOlbiofilm electrodes. Unlike the relatively featureless photocurrent profiles of the IOITOIPSII, profiles belonging to IO-ITOlbiofilm (Figure 4A) consistently show an initial spike, followed by a fast decay and a second slow steady rise in photocurrent. These profiles can be discussed in the context where it is known that cyanobacteria participate in quorum sensing, ${ }^{18}$ and that bacterial membranes can behave similarly to neuron membranes where depolarization, repolarization and hyperpolarization occur through the opening of gated ion channels. ${ }^{19}$ The photocurrent profile of IO-ITOlbiofilm is reminiscent of the latter phenomenon, and the initial spike may be explained by the opening of gated channels, perhaps triggered by intracellular light-induced reduction events. An efflux of ions or reduced redox active
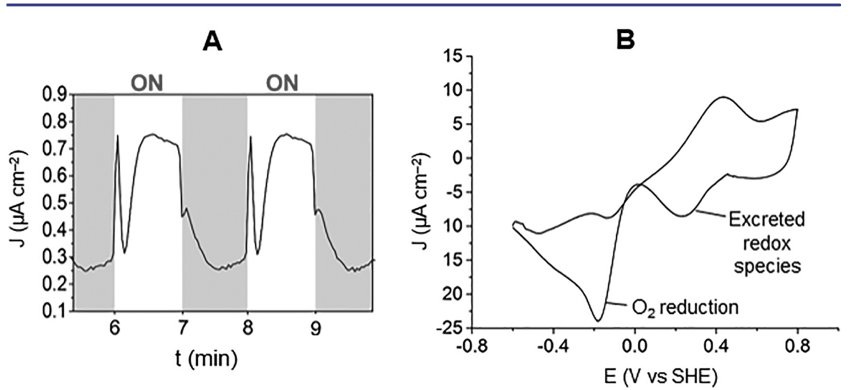

Figure 4. (A) Representative photocurrent profile of IO-ITOlbiofilm after $12 \mathrm{~h}$ light irradiation ( $E_{\text {app: }}: 0.3 \mathrm{~V}$ vs SHE). (B) Difference between $\mathrm{CV}$ scans $\left(\nu=10 \mathrm{mV} \mathrm{s}^{-1}\right)$ of IO-ITOlbiofilm under light irradiation and under dark-adapted anaerobic conditions. The CV scans obtained in the dark were subtracted from those obtained under light (Figure S12). CV scans were carried out under Ar purged conditions at $25{ }^{\circ} \mathrm{C}$, using a red light source $\left(\lambda_{685 \mathrm{~nm}}: 1 \mathrm{~mW} \mathrm{~cm}{ }^{-2}\right)$. 
species would follow, as detected by the electrode surface. As the stored ionic species rapidly depletes, the initial spike also rapidly declines. A second slower and more sustained rise in photocurrent follows, which may be due to the efflux of newly formed products from photosynthesis via the opened channels. Interestingly, the photoelectrochemical profiles of IO-ITOI biofilm change over 5 days (Figure S11), perhaps reflecting changes in the cellular physiological states or to the extracellular environment over time.

Cyclic voltammetry $(\mathrm{CV})$ scans were performed on the IOITOlbiofilm electrodes under dark (anaerobic) and light conditions. The CV scans obtained in the dark were subtracted from those obtained under light (Figure S12) to detect the release of any redox active species at the biofilm interface during light irradiation. A reversible redox wave can be observed with a midpoint potential of $0.34 \mathrm{~V}$ vs SHE (Figure $4 \mathrm{~B}$ ), which may correspond to benzoquinone or flavin derivatives. ${ }^{5}$ The reduction wave at $-0.2 \mathrm{~V}$ vs SHE was assigned to $\mathrm{O}_{2}$ reduction and provides evidence that the biofilm is active and evolving $\mathrm{O}_{2}$. The role of this light-triggered release of reduced species is unknown, but may be important for the maintenance of cellular function and osmotic pressure, serve as a self-protection mechanism against over-reduction of the electron transport system, ${ }^{20}$ and/or provide a means of multicellular communication. ${ }^{12}$

In conclusion, we have shown that the IO-ITO structure is a versatile electrode platform for supporting photoactive biological materials ranging from the nano to micrometer-scale. In this comparison study, we establish that the light-to-charge conversion output of the IO-ITOIPSII systems is initially greater than the IO-ITOlbiofilm system. However, the photoelectrochemical conversion rates (expressed by TOF) by PSII in vivo vs in vitro were found to be comparable. Hence, differences in output must partly be due to the higher photoabsorber/biocatalyst density present in the IO-ITOIPSII systems. As such, one simple way of enhancing biofilm photoelectrochemistry output would be to further optimize the electrode architecture for high-density cell loading. The addition of a quinone mediator (MET) was found to increase the photoresponse of the biofilm, but the effect was temporary and potentially cytotoxic. Despite the lower output, the IOITOlbiofilm system demonstrated impressive stability, overtaking the TON of IO-ITOIPSII after 2 days. Considering that the photoresponse of the biofilms was still increasing after 5 days with no signs of decline, the full longevity and electrogenic potential of the IO-ITOlbiofilm electrode has yet to be demonstrated. Lastly, we have provided novel mechanistic insights into the origins of the photoelectrogenic activity of Synechocystis biofilms. Our observations are consistent with an indirect ET pathway that involves the release of intracellular diffusional species. As such, we have demonstrated that such comparison studies are important for unraveling bottlenecks in photosynthetic biofilm systems, and for pointing the way toward higher performance.

\section{ASSOCIATED CONTENT}

\section{S Supporting Information}

The Supporting Information is available free of charge on the ACS Publications website at DOI: 10.1021/jacs.7b08563.

Materials and methods, Figures S1 to S12 and Tables S1 to S3 (PDF)

\section{AUTHOR INFORMATION}

\section{Corresponding Author}

*reisner@ch.cam.ac.uk

ORCID

Erwin Reisner: 0000-0002-7781-1616

Notes

The authors declare no competing financial interest.

Additional data related to this publication are available at the University of Cambridge data repository (https://doi.org/ 10.17863/CAM.13315).

\section{ACKNOWLEDGMENTS}

This work was supported by the EPSRC (DTA Ph.D. studentship to K.P.S.), an ERC Consolidator Grant "MatEnSAP" (682833 to E.R. and J.Z.), the Leverhulme Trust (to P.B and C.H.) and a Wolfson Merit Award from the Royal Society (to A.W.R.). We thank David J. Lea-Smith for recording the TEM image and Dr. Khoa H. Ly and Dr. Nikolay Kornienko for helpful discussions.

\section{REFERENCES}

(1) Barber, J.; Tran, P. D. J. R. Soc., Interface 2013, 10, 20120984.

(2) Suga, M.; Akita, F.; Hirata, K.; Ueno, G.; Murakami, H.; Nakajima, Y.; Shimizu, T.; Yamashita, K.; Yamamoto, M.; Ago, H.; Shen, J.-R. Nature 2015, 517, 99.

(3) Blankenship, R. E.; Tiede, D. M.; Barber, J.; Brudvig, G. W.; Fleming, G.; Ghirardi, M.; Gunner, M. R.; Junge, W.; Kramer, D. M.; Melis, A.; Moore, T. A.; Moser, C. C.; Nocera, D. G.; Nozik, A. J.; Ort, D. R; Parson, W. W.; Prince, R. C.; Sayre, R. T. Science 2011, 332, 805.

(4) Kato, M.; Zhang, J. Z.; Paul, N.; Reisner, E. Chem. Soc. Rev. 2014, 43, 6485 .

(5) McCormick, A. J.; Bombelli, P.; Bradley, R. W.; Thorne, R.; Wenzel, T.; Howe, C. J. Energy Environ. Sci. 2015, 8, 1092.

(6) Cereda, A.; Hitchcock, A.; Symes, M. D.; Cronin, L.; Bibby, T. S.; Jones, A. K. PLoS One 2014, 9, e91484.

(7) Sekar, N.; Jain, R.; Yan, Y.; Ramasamy, R. P. Biotechnol. Bioeng. 2016, 113, 675 .

(8) McCormick, A. J.; Bombelli, P.; Scott, A. M.; Philips, A. J.; Smith, A. G.; Fisher, A. C.; Howe, C. J. Energy Environ. Sci. 2011, 4, 4699.

(9) Quintana, N.; Van der Kooy, F.; Van de Rhee, M. D.; Voshol, G. P.; Verpoorte, R. Appl. Microbiol. Biotechnol. 2011, 91, 471.

(10) Mersch, D.; Lee, C.-Y.; Zhang, J. Z.; Brinkert, K.; FontecillaCamps, J. C.; Rutherford, A. W.; Reisner, E. J. Am. Chem. Soc. 2015, $137,8541$.

(11) Hasan, K.; Bekir Yildiz, H.; Sperling, E.; Conghaile, P. O.; Packer, M. A.; Leech, D.; Hägerhäll, C.; Gorton, L. Phys. Chem. Chem. Phys. 2014, 16, 24676.

(12) Borole, A. P.; Reguera, G.; Ringeisen, B.; Wang, Z.-W.; Feng, Y.; Kim, B. H. Energy Environ. Sci. 2011, 4, 4813.

(13) Bombelli, P.; Bradley, R. W.; Scott, A. M.; Philips, A. J.; McCormick, A. J.; Cruz, S. M.; Anderson, A.; Yunus, K.; Bendall, D. S.; Cameron, P. J.; Davies, J. M.; Smith, A. G.; Howe, C. J.; Fisher, A. C. Energy Environ. Sci. 2011, 4, 4690.

(14) Bombelli, P.; Müller, T.; Herling, T. W.; Howe, C. J.; Knowles, T. P. J. Adv. Energy Mater. 2015, 5, 1401299.

(15) Melis, A. Philos. Trans. R. Soc., B 1989, 323, 397.

(16) Fraser, J. M.; Tulk, S. E.; Jeans, J. A.; Campbell, D. A.; Bibby, T. S.; Cockshutt, A. M. PLoS One 2013, 8, e59861.

(17) Zhang, J. Z.; Sokol, K. P.; Paul, N.; Romero, E.; van Grondelle, R.; Reisner, E. Nat. Chem. Biol. 2016, 12, 1046.

(18) Sharif, D. I.; Gallon, J.; Smith, C. J.; Dudley, E. ISME J. 2008, 2, 1171.

(19) Prindle, A.; Liu, J.; Asally, M.; Ly, S.; Garcia-Ojalvo, J.; Süel, G. M. Nature 2015, 527, 59.

(20) Scheibe, R.; Dietz, K.-J. Plant, Cell Environ. 2012, 35, 202. 